\title{
Applicability of the GP Device to the Circle of Willis Arteries by Using a Mathematical Model
}

\author{
G. Romero, M.L. Martinez, J. Felez \\ ETSI Engineering, Technical University of Madrid \\ Madrid, Spain \\ e-mail: gregorio.romero@upm.es, \\ luisa.mtzmuneta@upm.es,jesus.felez@upm.es
}

\author{
G. Pearce, N.D. Perkinson \\ Wolverhampton University \\ Wolverhampton, United Kingdom \\ e-mail: gillpearce@googlemail.com, \\ neil@perkinson.org.uk
}

\begin{abstract}
According to the World Health Organization [1] , 15 million people suffer stroke worldwide each year; of these, 5 million die and 5 million are permanently disabled. Stroke is therefore a major cause of mortality world-wide. The majority of strokes are caused by a blood clot that occludes an artery in the brain, and although thrombolytic agents such as Alteplase are used to dissolve clots that arise in the arteries of the brain, there are limitations on the use of these thrombolytic agents. However over the past decade, other methods of treatment have been developed which include Thrombectomy Devices e.g. the 'GP' Thrombus Aspiration Device ('GP' TAD). Such devices may be used as an alternative to thrombolytics or in conjunction with them to extract blood clots in arteries such as the middle cerebral artery of the midbrain brain, and the posterior inferior cerebellar artery (PICA) of the posterior aspect of the brain. In this paper, we mathematically model the removal of blood clots using the 'GP' TAD from selected arteries of the brain where blood clots may arise taking into account factors such as the resistances, compliances and inertances effects. Such mathematical modelling may have potential uses in predicting the pressures necessary to extract blood clots of given lengths, and masses from arteries in the Circle of Willis - posterior circulation of the brain.
\end{abstract}

Keywords- Biomedical engineering, Thrombectomy Device, Circle of Willis, Simulation .

\section{INTRODUCTION}

According to research conducted by the World Health Organization [1], Cardiovascular diseases (CVDs) are the number one cause of death globally, more than from any other cause. It is estimated that more than 17 million people died from this cause in 2004, representing an important percentage of all global deaths (29\%); an estimated 5.7 million were due to stroke. Strokes are usually severe events and are mainly caused by a blockage that prevents blood from flowing to the brain. The most typical reason for this blockage is a build-up of fatty deposits on the inner walls of the blood vessels that supply the brain. Strokes can also be caused by bleeding from a blood vessel in the brain or from blood clots.

The Circle of Willis (fig. 1) supplies blood to the brain. It is divided into an anterior aspect of the Circle of Willis and a posterior aspect. The posterior aspect supplies the posterior parts of the brain including the cerebellum, and occipital areas. It is not uncommon for strokes that affect these parts of the brain to produce symptoms such as lack of coordination, visual disturbances and balance problems. The vertebral arteries join to form the basilar artery from which joins the Circle of Willis at the posterior aspect of the brain. Smaller arteries branch off these arteries to supply the different aspects of the hind brain.

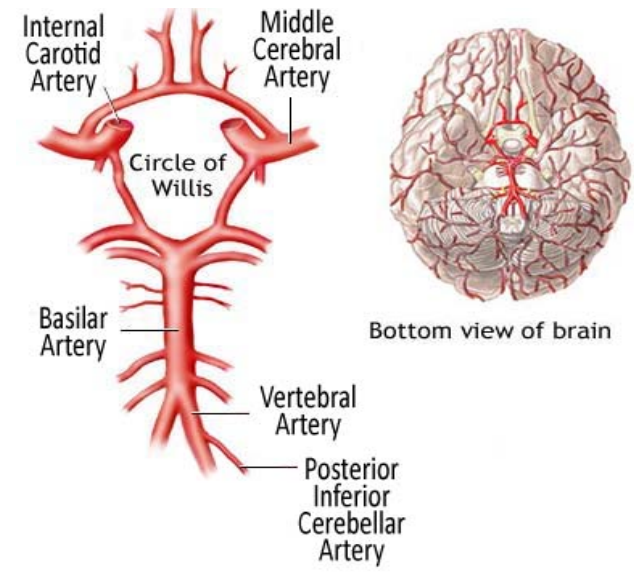

Figure 1. Schematic representation of the Circle of Willis. [2]

Recently thrombolysis has been used to dissolve clots in the posterior (as well as the anterior) circulation of the brain. However as with the use of thrombolytics in the anterior part of the brain, the same constraints apply to its use. Due to the risk of hemorrhage it is contraindicated in patients who have undergone recent surgery. Therefore it is useful to have more than one treatment method available and in the last decade the use of mechanical clot retrieval devices has increased.

During the last decade Thrombectomy Devices have become more widely used and have been developed as an alternative and more mechanical means for clot removal. The need to study new Thrombectomy devices like the one described here means that a computer pre-modelling may be helpful in the optimization and fine-tuning of such devices prior to clinical trials.

In the case of this study, the device 'GP' TAD has been designed to remove blood clots without the need to make contact with the clot itself, which potentially reduces the risk 
of problems such as downstream embolisation. The extraction of the obstructing element (i.e. the blood clot) by the device is done by means of vacuum suction provided by the action of a pump. To do this, an increasing pressure is applied over time until a value is attained at which the clot is captured. The force required to separate the clot from the arterial wall is analysed by modelling a complianceresistance system to represent the interaction between both parts. A viscous damper will be used that allows us to consider the change from static to dynamic friction in its relative movement. To implement this we define different sections of the mechanism studied in the paper. Blood is deemed to be an incompressible fluid, while the tube between the pump and the 'GP' device is deemed to be nonelastic. The loss load will be due mainly to the existent flow, which by means of previous studies has been shown to be laminar.

Our model is applied to the use of the 'GP' TAD to the posterior arteries of the brain (PICA, BA and VA), to investigate the potential use of such a device and to investigate the use of such modeling as a predictor of design performance. The main objective of the development of this simulation model is to analyze the minimum pressure required to perform clot extraction using one fixed adhesion force between the clot and the artery wall. It is important that both the suction pressure applied and the time required to complete the clot extraction are reasonable for use in clinical situations, and are consistent with any experimentally obtained data. The presented study has been applied for a range of lengths and occlusions of blood clot.

\section{GP' TAD DEVICE STRUCTURE}

The 'GP' TAD device (fig. 2) [3] action involves using a vacuum pump that provides the necessary suction pressure for the operation, joined to a very long catheter $(\approx 1 \mathrm{~m})$. The 'GP' device is located at the end of this catheter. The internal surface of the 'GP' device has been mathematically designed to optimize the distribution of forces. It is also associated with low forces at the periphery of the device which may therefore reduce the risk of arterial collapse during aspiration of the clot $[4,5]$.

The proposed procedure for using this device is to introduce it into an artery (via the arterial catheter) in close proximity to the occluding blood clot, and to position it at a distance of approximately $3 \mathrm{~mm}$ from the clot. Then the suction pressure is slowly increased until the clot cross the 3 $\mathrm{mm}$ that separates it from the 'GP' device and clot capture therefore occurs; at his time, the device and the clot would be removed from the body easily.

The device is currently being developed as a potential thrombus aspiration device through a series of in vitro studies. This device has the potential to be used in relatively small arteries. It has no moving parts and therefore should reduce the risk of breakage in a vessel. Since it does not touch the clot itself it also has the potential to reduce the risk of clot disruption (downstream embolisation).

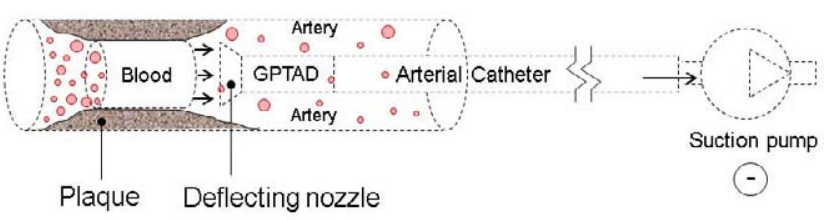

Figure 2. Schematic view of the 'GP' Thrombus Aspiration Device.

The 'GP' TAD has been modelled in previous studies using different simulation software packages [6], and different existing techniques have been considered for the modelling and simulation [7]. Some methods which have been considered previously for their applicability are the Boltzmann flow simulation technique, and finite elements modelling and its implementation in Matlab (C) software, with 2D or 3D models; or by means of Laplace transformations using Dynamic Motion Solver (C) software.

The objective of this study is to obtain the optimal mathematical model for use in demonstrating the potential performance of the 'GP' TAD device under different conditions of blood flow and size of blood clot, in a given vessel.

\section{MATHEMATICAL MODELLING}

This section gives a brief description of the device under study, as well as the parts comprising it. We describe the model used for the simulation, the phenomena considered, and the values of the parameters used are described.

The vacuum pump creates the necessary suction pressure to carry out the extraction, and is positioned at the other end of the catheter that does not contain the 'GP' TAD.

If we analyze the schematic view (fig. 2), we can view the catheter, 'GP' device and artery as a pipe of different parameters. It is necessary to analyze the lost of pressure due to the mass of fluid, the compressibility that the blood and artery are subjected to, and finally the resistances that appear when fluid and blood clot flow into the pipe [8].

In the model, the suction pump can be represented by a variable pressure ' $\mathrm{P}$ ' whose value will increase from zero to a non-determined value $(0-[-2,-100] \mathrm{kPa})$ suitable for carrying out the operation of clot removal. The value of ' $\mathrm{P}$ ' will be obtained from the optimization of the developed model. The time taken to reach the maximum value of pressure has been obtained from experiment and is about 3 sec., after which time the pressure provided by the pump remains constant.

To generate the correct model, it is necessary firstly to analyze the flow variation taking into account the pressure lost that appears in the different parts of the system.

When a fluid goes into a pipe from point 1 to point 2 , it is necessary to overcome the inertance of the fluid. If we consider that the fluid is not compressible and the pipe as a non elastic element with no deformations, we can assume that the flow ' $Q$ ' is constant across the pipe. In addition, the 'momentum' associated with the inertances can be defined as the product of the inertance value and the flow.

$$
m_{p}=I \cdot Q
$$


On the other hand, if we consider that a force is equal to the fluid mass multiplied by the acceleration of it, which is corresponding with the derived velocity of the fluid $(\mathrm{Q} / \mathrm{A})$, we have that:

$$
P_{1} \cdot A-P_{2} \cdot A=(\rho \cdot A \cdot L)(\dot{Q} / A)
$$

, where ' $\mathrm{P}_{1}$ ' and ' $\mathrm{P}_{2}$ ' are the existent pressure in two points of a pipe, ' $\rho$ ' is the density of the fluid, ' $A$ ' the section pipe, and ' $L$ ' the length between the two points 1 and 2 .

Simplifying previous equation, we can conclude that:

$$
\Delta P=\rho \cdot L \cdot \frac{\dot{Q}}{A}
$$

On the other hand, the 'momentum' associated to the inertances (eq. 1) can be defined additionally as:

$$
m_{p}=\int^{t} \Delta P \cdot d t
$$

By replacing eq. (3) into eq. (4), we can write that:

$$
m_{p}=\int^{t} \rho \cdot L \cdot \frac{\dot{Q} \cdot d t}{A}=\rho \cdot L \cdot \frac{Q}{A}
$$

Finally, if we compare eqs. (1) and (5) we can conclude that the inertance corresponding with a fluid flowing into a pipe can be written as:

$$
I=\rho \cdot L / A
$$

Once defined the effect corresponding with the inertance effect of a fluid flowing into a pipe can be evaluated, and we can define the different equations that are associated with the first section of the schematic view as shown in the following figure.

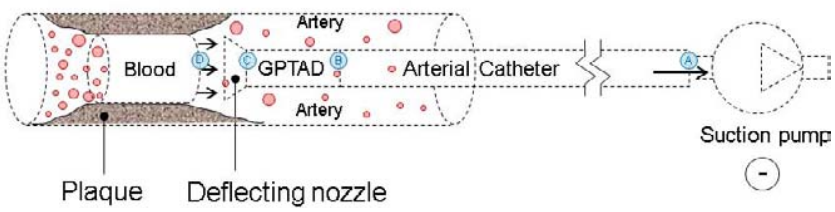

Figure 3. Schematic view of the 'GP' Thrombus Aspiration Device.

If we take into account the equation (3), the derived flow of the fluid that goes into the catheter can be defined as the pressure difference between beginning and end points divided by the inertance of the fluid (blood). The pressure lost is not only the difference of pressure between point $\mathrm{A}$ (depressure pump) and B, but the effect of the linear load loss ' $R$ ' due to the friction between the liquid particles and the catheter walls, taking the next expression:

$$
\frac{d Q_{\text {catheter }}}{d t}=\left(\frac{A_{\text {catheter }}}{\rho \cdot L_{\text {catheter }}}\right) \cdot\left(P_{\text {Pump }}-P_{B}-R_{\text {catheter }} \cdot Q_{\text {catheter }}\right)
$$

To simplify, only linear load losses have been taken into account. If we suppose that the catheter is composed of successive horizontal pipes of constant cross section in each section, the load loss is reduced to a pressure loss as the fluid advances along the catheter, the loss being progressive and proportional to the length of the catheter. If we assume that the blood flow is laminar due to the Reynolds number being approximately $1.000(\operatorname{Re}<2.200)$, the equation that governs the behaviour in the catheter can be determined by the following expression:

$$
R=\frac{128 \cdot \eta \cdot L}{\pi \cdot D^{4}}
$$

, where ' $\eta$ ' is the viscosity of the fluid, and ' $D$ ' the diameter of the catheter. Some studies show that in diseased arteries the viscosity of the blood varies, increasing its value but for this first approximation we used a normal value of 0.0035 $\mathrm{Pa} \cdot \mathrm{s}$.

In the same way, the resulting flow of blood that goes into the 'GP' device can be defined as the pressure difference between beginning and end points divided by the inertance of the content blood:

$$
\frac{d Q_{G P}}{d t}=\left(\frac{A_{G P}}{\rho \cdot L_{G P}}\right) \cdot\left(P_{B}-P_{C}-R_{G P} \cdot Q_{G P}\right)
$$

In addition, since the artery is located near the end of the 'GP' TAD, it is necessary to consider the transition between both elements as a secondary load loss caused by the deflecting nozzle due to the difference in diameter of the 'GP' device and the artery respectively. The latter can give rise to subsequent variations in flow. These load losses can be represented as a resistance and can be calculated as a function of the mean value $\left(\mathrm{D}_{\mathrm{m}}\right)$ between the diameter of the catheter and the diameter of the artery, and the load loss coefficient $(\xi)$ which is a dimensionless parameter that quantifies the loss produced and depends on the geometry of the deflecting nozzle, with the following expression:

$$
R_{\text {nozzle }}=8 \cdot \rho \cdot \xi \cdot \frac{Q_{\text {artery }}}{\pi^{2} \cdot D_{m}{ }^{4}}
$$

, where ' $Q_{\text {artery }}$ ' is the flow which circulates in the section between the end of the 'GP' device and the artery.

Taking this into account, (with respect to the tip of the 'GP' and the part of artery that goes from this point to the blood clot), we can define the derived equation including the effect of the linear load loss due to the friction between the liquid particles and the artery walls, and the secondary losses caused by the deflecting nozzle:

$$
\frac{d Q_{\text {artery }}}{d t}=\left(\frac{A_{\text {artery }}}{\rho \cdot L_{\text {artery }}}\right) \cdot\left(P_{C}-P_{D}-\left(R_{\text {nozzle }}+R_{\text {artery }}\right) \cdot Q_{\text {artery }}\right)
$$

Once these equations have been defined in the model, it is necessary to change from the hydraulic to the mechanical domain before considering the clot element, to be able to evaluate the clot extraction taking into account the physical friction between the clot and the artery.

To formulate the corresponding equations from the different described phenomena, it is necessary to assume that the flow after and before resistances and inertances are the same. 


\section{Clot Mathematical Modelling}

Accurately defining the clot model in order to model it is the most complex part of the model. In the case of a $100 \%$ blood clot occlusion, including the existence of plaque or not, we can in both cases approximate the clot as a cylindrically-shaped element of [0.5-5] cm long, and of a mass that falls between [0.5-0.1] gram. This element has usually formed in another location, usually in a vascular artery, and has become dislodged remaining trapped in smaller diameter arteries, such as the Circle of Willis arteries considered in this work. Therefore, due to this difference in diameter, the clot becomes attached to the wall by a force of adhesion that needs to be overcome in order to begin movement of the clot for its removal. In addition, the relative movement between the clot and the artery presents static and dynamic friction, which needs to be taken into account. If a correct approximation to reality is to be achieved all these phenomena, as well as the circumstances restricting clot movement, need to be considered.

As already stated, the phenomenon preventing clot movement is the difference in diameter between the clot and the artery where it is located. Experimental data demonstrated that low times of residence of the clot in the artery, the value of the adhesion force is $0.01 \mathrm{~N}$, which value will be considered.

In order to decide when to begin the movement of the obstructive element, firstly we need to insert a spring ' $\mathrm{K}_{\text {clot }}$ ' into the model to measure the force supported by the beginning of the clot and its deformation.

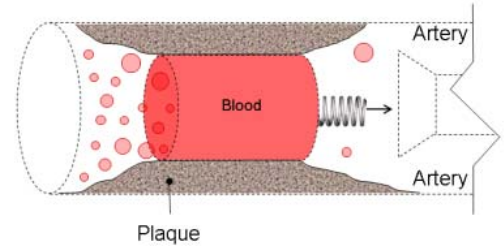

Figure 4. Force supported by the beginning of the clot.

To know when the force will be reached in this equivalent spring and, therefore, when the clot movement will begin, it is essential to calculate the displacement of the spring when it is subjected to $0.01 \mathrm{~N}$ through a typical spring equation. Therefore, only when the spring undergoes this displacement should the clot be allowed to move; to the contrary it would be prevented.

Secondly, the friction between the clot and the arterial wall creates another resistance factor. The value of this parameter must be variable depending on whether the clot has not begun its movement (static friction) or if it is already moving (dynamic friction), so that when the clot begins to move the friction value will drop considerably. This value is obtained starting from the Stokes equation and can be given a value of $2.5 \cdot 10^{-6} \mathrm{~N} \cdot \mathrm{s} / \mathrm{m}$ for the static friction and one order of magnitude lower than for the dynamic friction. The transition between both values marks the beginning or end of the clot movement by means of the displacement of the spring previously defined ' $\mathrm{K}_{\text {clot }}$ '. So, when the displacement undergone by this spring is less than that calculated, static friction will predominate; to the contrary, if it is greater, then the friction will be dynamic.

Additionally, to ensure that the clot remains at rest while the force existing at its beginning is less than the adhesion force, a spring-damper system joined to a wall must be used $\left(\mathrm{K}_{\mathrm{wall}}\right.$ and $\left.\mathrm{R}_{\mathrm{wall}}\right)$. In this system, while the clot doesn't receive the force of minimum suction (that would cause it to move), it has a zero speed. However, when it begins to move, the spring-damper system must be cancelled allowing its extraction.

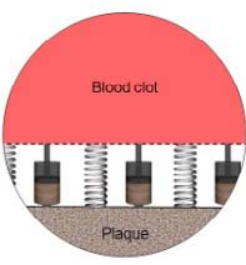

a).

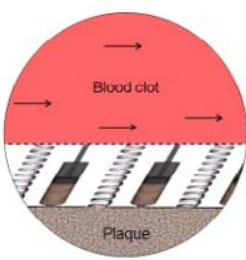

b).

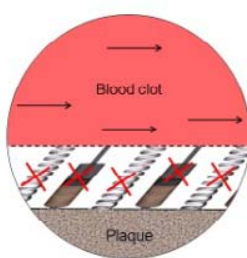

c).
Figure 5. Detailed view of contact blood clot - artery/plaque wall. a). $\left.\left.F_{\text {suction }}=0.0 \mathrm{~N}, \mathrm{~b}\right) . \mathrm{F}_{\text {suction }}<0.01 \mathrm{~N}, \mathrm{c}\right) . \mathrm{F}_{\text {suction }}>0.01 \mathrm{~N}$

Therefore, while the force representing the deformation of the clot is lower than the adhesion force, it will remain attached to the wall, thereby preventing any movement. However, if the force exceeds the value of the adhesion force the spring-damper system will be eliminated with the clot becoming free and moving in accordance with the suction pressure acting on it from the system, allowing it to be removed. The elimination of the spring-damper system will be performed from the displacement of the spring representing the clot deformation, as was seen in the condition imposed on the static/dynamic friction. After different experiments, the optimum values of this springdumper are of $10^{10} \mathrm{~N} / \mathrm{m}$ and $10^{9} \mathrm{~N} \cdot \mathrm{s} / \mathrm{m}$ respectively.

In addition to the spring and the resistance inserted, the model must have an inertance element that represents the mass of the clot ' $\mathrm{M}_{\text {clot }}$ '.

After previous arguments, we can define the decceleration of the clot according to pressures and the values of the spring and dampers as eluded to above in the modelling. We take into account the necessity to change from the hydraulic to the mechanical domain. This is necessary to introduce the area of the clot multiplying the pressure before the clot. Finally, the equation can be generated as the sum of the force associated with the pressure existing near the clot ' $\mathrm{P}_{\mathrm{D}}$ ' (fig. 3), and the forces associated with the resistances and compliances, taking the necessary signs into account.

$$
\frac{d V_{c l o t}}{d t}=\frac{A_{c l o t} \cdot P_{D}-\left(R_{\text {wall }}+R_{c l o t}\right) \cdot V_{c l o t}+K_{\text {wall }} \cdot X_{K_{\text {wall }}}}{M_{\text {clot }}}
$$

\section{COMPRESSIBILITY PHENOMENA}

In a pipe, the fluid acts as a compliance producing a decrease in volume when the pressure required for compression is increased, and it is necessary to take into account the blood compressibility. In our case, we can define this behavior associated with the blood as dependent on the Bulk's blood coefficient and section of the artery. If we 
assume a circular geometry, it can be defined as a capacitance of ' $\mathrm{K}_{\text {blood }}$ ' value:

$$
K_{\text {blood }}=4 \cdot B / \pi \cdot D_{a}{ }^{2} \cdot L
$$

In addition, it is necessary to take into account the compressibility of the pipe material and not only the fluid. Although we can assume that catheter and 'GP' device are incompressible due to the high rigidity, it is necessary to insert the compressibility of the length of artery that appears between the 'GP' device and the clot, in accordance with its Young's modulus (E):

$$
K_{\text {material }}=\frac{E \cdot h}{V_{0} \cdot 2 \cdot r_{0}}
$$

where ' $\mathrm{V}_{0}$ ' is the artery initial volume and ' $\mathrm{r}_{0}$ ' is the artery initial radius.

From consideration of both compressibility phenomena, in addition to the system composed with equations (7), (9), (11) and (12) it is necessary to introduce other derived equations to obtain the different flows from the volumes and pressures associated with all these various entities.

$$
\begin{aligned}
& \frac{d P_{\text {catheter }}}{d t}=K_{\text {fluid_catheter }} \cdot\left(Q_{\text {catheter }}-Q_{G P}\right) \\
& \frac{d P_{G P}}{d t}=K_{\text {fluid_GP }} \cdot\left(Q_{G P}-Q_{\text {artery }}\right) \\
& \frac{d P_{\text {artery }}}{d t}=K_{\text {eq_artery }} \cdot\left(Q_{\text {artery }}-A_{\text {artery }} \cdot V_{\text {clot }}\right)
\end{aligned}
$$

, where ' $\mathrm{K}_{\text {eq_artery }}$ ' is the equivalent compliance due to the combination of three compressibilities (fluid artery, material of artery and deformation of the clot due to the suction force). We therefore apply the necessary domain changes by using the clot area $\left(\mathrm{A}_{\text {clot }}\right)$.

$$
K_{\text {eq_artery }}=\frac{K_{\text {fluid_artery }} \cdot K_{\text {artery }} \cdot K_{\text {clot }}}{K_{\text {artery }} \cdot K_{\text {clot }}+K_{\text {fluid_artery }} \cdot K_{\text {clot }}+A_{\text {clot }}{ }^{2} \cdot K_{\text {fluid_artery }} \cdot K_{\text {artery }}}
$$

Finally, we add in the relationship for the displacement of the spring system joined to the wall to ensure that the clot remains at rest, such that the force existing at the beginning of the analysis is less than the adhesion force and can be written in the form:

$$
\frac{d X_{K_{\text {wall }}}}{d t}=-V_{\text {clot }}
$$

With this system of equations, the mathematical model is complete and we can begin to determine the necessary times associated with the different pressures and values of parameters.

\section{RESULTS}

To obtain the simulation of the developed equations, DASSL algorithm will allows obtaining the evolution of the characteristic parameters of the model as well as letting them be compared.
The aim of this simulation is to compare the extraction of a clot in the VA, BA and PICA arteries considering different clot lengths and $70 \%$ occlusion. The use of the different arteries will affect directly to the used diameters into the model and the 'GP' and catheter sizes in the next way:

$$
\begin{array}{llll}
\text { - VA: } & D_{\text {artery }}=5,50 \mathrm{~mm} & \rightarrow & D_{G P}=D_{\text {catheter }}=5,0 \mathrm{~mm} \\
\text { - BA: } & D_{\text {artery }}=3,17 \mathrm{~mm} & \rightarrow & D_{G P}=D_{\text {catheter }}=3,0 \mathrm{~mm} \\
\text { - PICA: } & D_{\text {artery }}=2,50 \mathrm{~mm} & \rightarrow & D_{G P}=D_{\text {catheter }}=2,0 \mathrm{~mm}
\end{array}
$$

In the previously defined values, the standard elements ('GP' and catheter) would have normalized values, being necessary to choose the nearest diameter value to be inserted into the artery. The existence of different clot lengths will affect the mass of the clot being removed, and increase the time taken for clot removal as the value of the clot length increases Finally, an arterial occlusion of $70 \%$ will modify the area in which the suction force acts, considering that the clot reduces the cross-sectional area of the artery.

The following figures show the time taken to create the necessary suction force to move the clot across the $3 \mathrm{~mm}$ gap between the clot and 'GP' device, for different pump suction pressures, diameters and clot lengths.

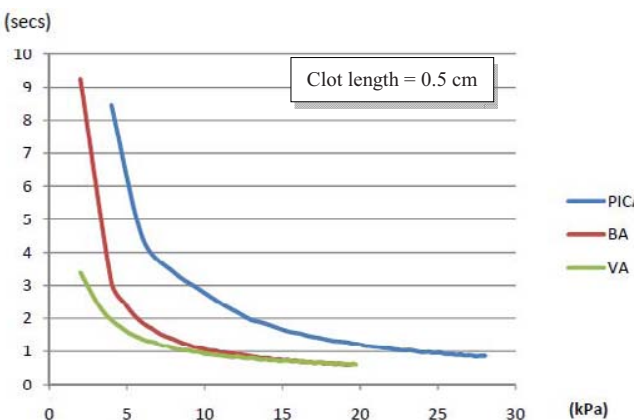

(secs)

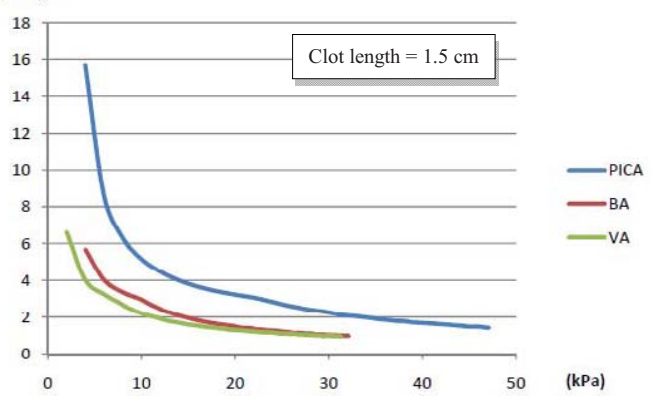

(secs)

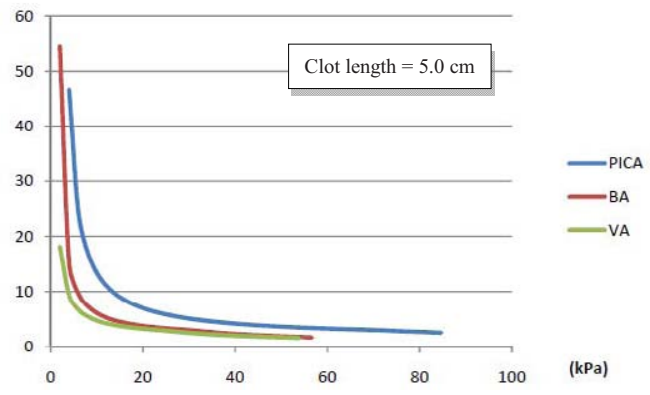

Figure 6. Pressure vs time required to clot removal. 
We observe that there is a difference on applying vacuum suction in each artery and for different clot lengths. The time taken to remove the blood clot in each instance is also different for each case. There are clearly differences in the times taken to remove the blood clot of various different lengths. While for a pressure of $-30 \mathrm{Kpa}$ the required time is below 5 seconds in all cases, the use of smaller suction pressures causes the range of times for clot removal to increase to 1 minute in some cases, e.g. with $-2 \mathrm{kPa}$ pressure. In some instances, the previous $-2 \mathrm{kPa}$ pressure sometimes increases the required time to more than 10 minutes -these cases correspond with the smaller arteries e.g. the PICA. It should be noted that the pressure losses are inversely related to the artery cross-section. It is possible to speculate that a minimum required suction pump pressure must be around $4 \mathrm{kPa}$ or $-6 \mathrm{kPa}$, and that the corresponding clot removal times would then take less than $3 / 4$ or $1 / 2$ minute. Higher clot removal times result when the clot lengths are greater e.g. with a $5 \mathrm{~cm}$ clot length in the PICA artery, (which is one of the smallest arteries).

For a suction pressure of $-4 \mathrm{kPa}$, the following figure shows how the time needed for clot removal increases as the diameter of the artery decreases. However in the case of one artery, the time taken for clot removal increases even when the clot length increases also.

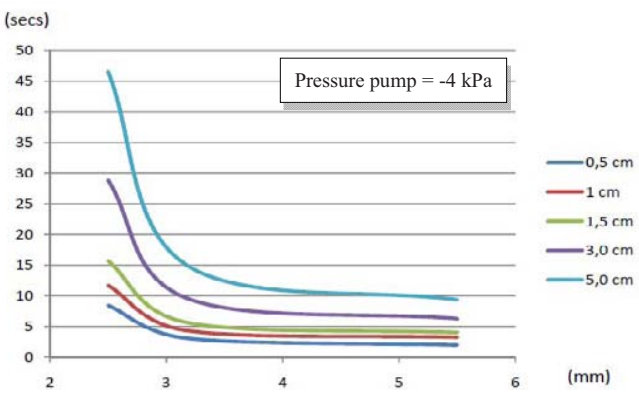

Figure 7. Diameter artery vs time required to clot removal.

This figure correlates with previous analysis completed for the Middle Cerebral Artery (MCA) which was also consistent with experimentally obtained data. In the case of the MCA artery, the diameter chosen for the 'GP' device and the catheter was $1 \mathrm{~mm}$. The corresponding times for clot extraction for a $5 \mathrm{~cm}$ clot $\approx 2$ minutes to using a suction pressure of $40 \mathrm{kPa}$.

These results indicate the importance of carrying out clot extraction as quickly as possible, and indicate the potential uses and importance of such modelling.

\section{CONCLUSIONS}

The development of this simulation model enables us to obtain an estimate of the minimum pressure required to perform the extraction of blood clots of different sizes and at different locations in the arteries of the Circle of Willis. In all cases we have taken the same age for the clot residing in the artery and the same values for the force of adhesion. This modelling has shown that the suction pressure together with the time required to complete clot removal are reasonable, and should be within acceptable clinical boundaries for any eventual potential use of the 'GP' TAD device in patients. Our results are similar to in-vitro experimentally obtained data. A similar analysis $[7,9]$ undertaken for its use in the MCA or the Popliteal artery enables further comparisons to be made. We compare the results of simulation modelling to measurements made in laboratory plastic tube models of arteries of comparable diameter where the 'GP' TAD was applied to successfully extract abattoir porcine blood clots. The suction pressures required for such clot extraction in these plastic tube models compare favourably with those predicted by the simulation modeling. We conclude therefore that the mathematical modelling presented may potentially be useful in predicting the performance of the GPTAD and could possibly be used in optimising the design of the device.

Our modelling indicates that blood clots in arteries in the Circle of Willis presented could potentially be extracted. Our modelling can be used to predict the suction pressures and times taken for clot removal. A clot of length typically $5 \mathrm{~cm}$, causing $70 \%$ occlusion of the artery (where $30 \%$ of the occlusion is due to plaque in the artery) requires suction pressures of around $6 \mathrm{kPa}$, to remove the clot and the time taken for clot extraction would typically take 30 seconds.

\section{FUTURE WORKS}

The presented mathematical modelling is now being implemented to model and simulate different adhesion forces and a range of percentage arterial occlusions. In this future modeling include the systolic/diastolic pressure effects for case of arterial occlusion below 100\% (including clot and plaque).

\section{REFERENCES}

[1] World Health Organization Website. http://www.who.int/mediacentre /factsheets/fs317/en/. Fact sheet $\mathrm{N}^{\circ} 317$. January 2011.

[2] U.S. National Library of Medicine Website. http://www.nlm.nih.gov/ medlineplus/ency/imagepages/18009.htm

[3] TA122 Alteplase for the treatment of acute ischaemic stroke: guidance (2007) National Institute for clinical Excellence.

[4] Pearce G, and Perkinson ND, "Biomechanical Probe". 2006; International Patent Corporate Treatise (WO2006120464) published 2006-11-16; European patent (EP1893195 (A2)) published 2008-0305; Japanese patent (JP2008639924 (T)) Published 2008-11-20; Chinese patent (CN101208049 (A)) published 2008-06-25.

[5] Pearce G, Patrick JH, Perkinson ND (2007). A new device for the treatment of thromboembolic strokes". Journal of Stroke and Cerebrovascular Diseases. 16(4):167-172.

[6] Pearce, G. et al. 2009. "An Investigation of fluid flow through a modified design for the "GP' device". 11th International Conference on Computer Modelling and Simulation, pp. 191-195. Cambridge, UK.

[7] Romero, G. et al. 2010. "Computational Modeling of a New Thrombectomy Device for the Extraction of Blood Clots". Advances in Computational Biology, series Advances in Experimental Medicine and Biology, AEMB, Vol. 680. Ed. Springer.

[8] Karnopp, D.C. et al. 1990. "System Dynamics: A Unified Approach". John Wiley \& Sons, Inc., Second edition.

[9] Pearce, G. et al. 2010. "Bond graph modelling of the in vitro performance of the GP thrombectomy Device in combination with local delivery of alteplase". International Journal of Stroke, Vol. 5, Issue Supplement s3, pag. 16. Special Issue. 